Advances in Radio Science (2004) 2: 289-291

(C) Copernicus GmbH 2004

\title{
A wonderful laboratory and a great researcher
}

\author{
N. M. Sheikh \\ Faculty of Electrical Engineering, University of Engineering and Technology, Lahore, Pakistan
}

\begin{abstract}
It was great to be associated with Prof. Dr. Karl Rawer. He devoted his life to make use of the wonderful laboratory of Nature, the Ionosphere. Through acquisition of the experimental data from AEROS satellites and embedding it with data from ground stations, it was possible to achieve a better empirical model, the International Reference Ionosphere. Prof. Dr. Karl Rawer has been as dynamic as the Ionosphere. His vision about the ionospheric data is exceptional and has helped the scientific and engineering community to make use of his vision in advancing the dimensions of empirical modelling. As a human being, Prof. Dr. Karl Rawer has all the traits of an angel from Heaven. In short he developed a large team of researchers forming a blooming tree from the parent node. Ionosphere still plays an important role in over the horizon HF Radar and GPs satellite data reduction.
\end{abstract}

\section{Introduction}

It is a great honour for me to be a student of Prof. Dr. Karl Rawer. I worked with him for two years from 1976 to 1978 at Institute of Space Research (IPW) Freiburg as DAAD Scholar. Before coming to Germany, I had worked on modelling and channel characterization of Meteor Forward Scattering Burst Communication (Akram et al., 1977) and had the intention of continuing the work in that area. However with the availability of the huge amount of the ionospheric data available from AEROS satellites (Sheikh et al., 1978a), he motivated me to work in data reduction and modelling of the ionosphere, a wonderful laboratory provided by nature. I enjoyed working with Prof. Dr. Karl Rawer and found him to be a dedicated and excellent researcher. Prof. Dr. Karl Rawer knew the ins and outs of the ionosphere and expressed the concepts so beautifully to his students that one felt as if one is travelling through the ionosphere. His presentation of both physical and empirical models was so clear that one

Correspondence to: N. M. Sheikh

(adstec@mailcity.com) could imagine looking at the global holographic images of the ionosphere.

I started with an ALGOL program provided by Prof. Dr. Karl Rawer and extended it to achieve results which could be used as a useful input to the International Reference ionosphere (IRI). The computational environment was still a giant Telefunken Computer with paper tape readers and magnetic tape mass storage medium. The things had already started changing in the form of networked PCs as I left. The technological changes have enabled faster and compact computational and storage facilities enabling the worldwide access of information on the web. Nevertheless fundamental concepts of modelling of the ionosphere have not changed drastically, though refinements have been incorporated.

\section{Spatial and temporal Resolution}

Large amounts of ground station data analyzed for several years month by month resulted in CCIR model (Sheikh et al., 1978a) giving monthly median values for $f o \mathrm{~F} 2$ and M (3000)F2. However due to the non-uniform spatial distribution of sounding stations, gaps of measurements existed specially over the oceans. Density measurements executed aboard satellites provided better global coverage. However the temporal resolution for a given satellite was limited. The Impedance Probe (IP) experiment aboard the AEROS-B satellite provided data with a spatial resolution of $48 \mathrm{~km}$ (averaging over six consecutive points), over an altitude of $230-880 \mathrm{~km}$ for two fixed local times. In our studies the Bent's model (Bent and Llewewllyn, 1970) was used for height reduction to evaluate the peak electron density. The latitudinal and longitudinal behaviour of $f o \mathrm{~F} 2$ was analyzed using AEROS data and interesting results were obtained. Pronounced variations in peak densities were observed in southern hemisphere different from the predictions of models that are based on ground station data Sheikh et al., 1978b). 


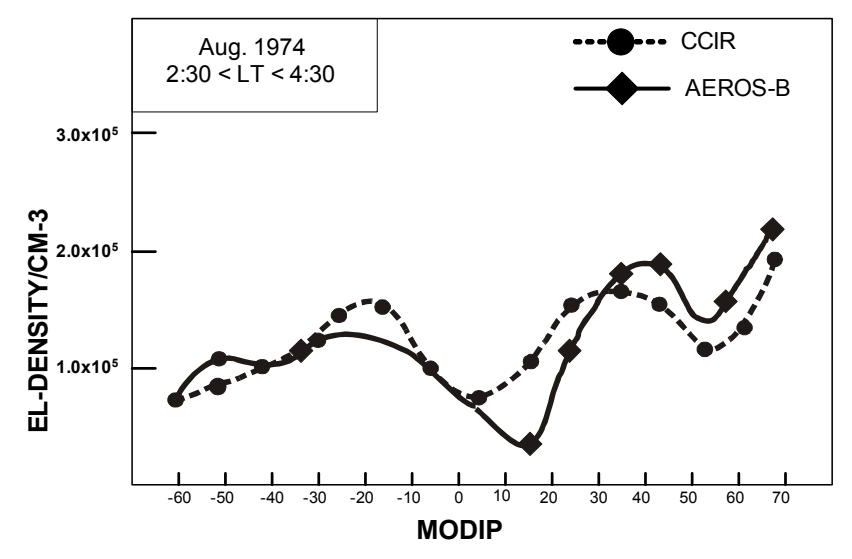

ATLANTIC

NIGHT

Fig. 1. Night-time Latitudinal variations of Electronic Density over Atlantic Ocean.

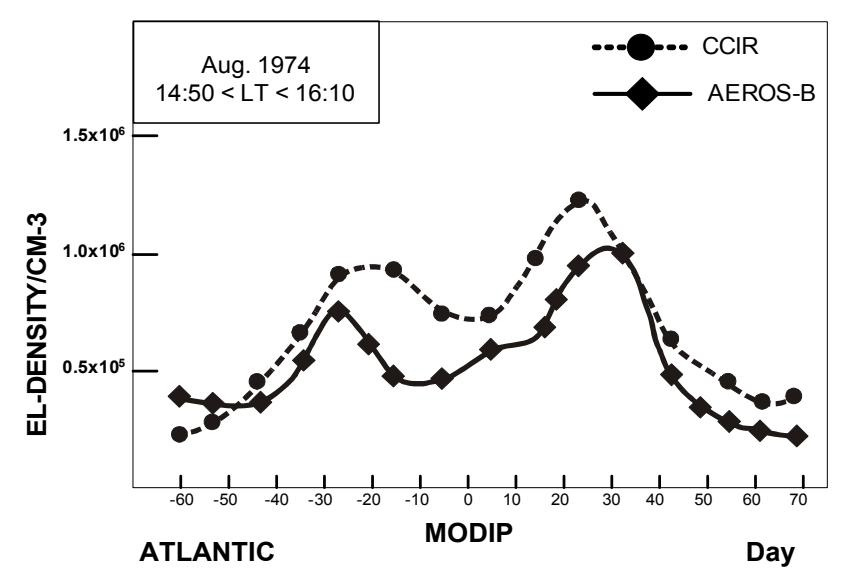

Fig. 2. Day-time Latitudinal variations of Electronic Density over Atlantic Ocean.

\section{Results}

From the work the following conclusions were drawn:

1. The overall agreement between ground data and satellite data models seemed to be good. The positions of the equator anomaly and the troughs are identical in most cases, except for the Atlantic Ocean where the equatorial trough shifted southwards by day and northwards by night (Figs. 1, 2, 3).

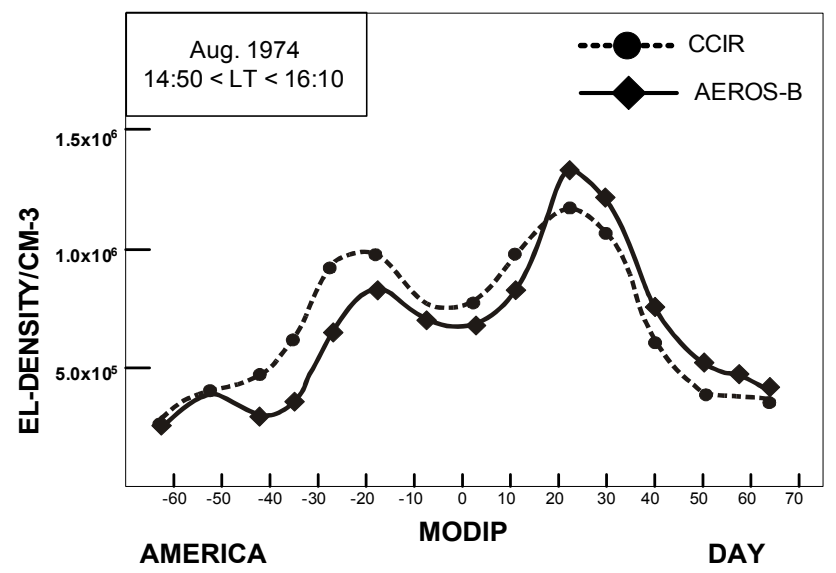

Fig. 3. Day-time variations of Electronic Density over America.

2. The latitudinal ionization gradients associated with the equatorial anomaly are actually higher than predicated by ground data models.

3. The longitudinal variations in ground data models are much smoothed out indicating that the coefficients in the model describe primarily a universal time effects rather than a true longitudinal effect. Longitudinal relationship is shown in polar form in Fig. 4. For winter time in the southern hemisphere, both CCIR model and AEROS data give smaller electron density values. However CCIR data is over smoothed and maximum is shifted slightly.

4. In ground data based model both hemispheres are not equally well represented. This may be due to the smaller number of inputs from southern ionospheric stations available.

\section{Conclusions}

Through the improvements in the ionospheric models using satellite data, a better model is available on the web and can be used easily for applications in scientific and engineering applications. 


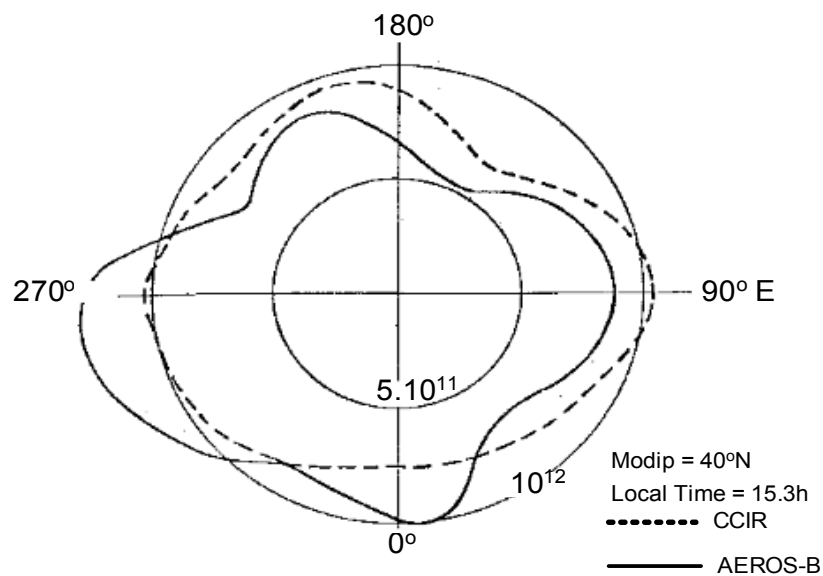

Fig. 4a. Longitudinal Variations of Electron Density in Northern hemisphere at modip $=40^{\circ} \mathrm{N}$.

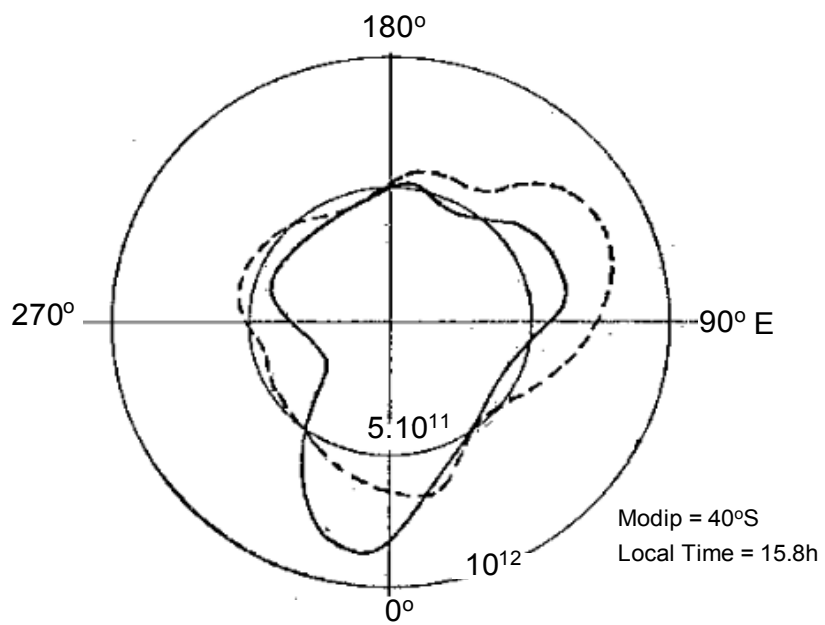

Fig. 4b. Longitudinal Variations of Electron Density in Southern hemisphere at modip $=40^{\circ} \mathrm{S}$.

\section{References}

F. Akram, Sheikh, N. M., Javed, A., and Grossi, M. D.: Impulse Response of a meter Burst Communication Channel Determined by Ray-Tracing Techniques, IEEE Trans. on Comm., Com-25 No. 4, 467-470, 1977.

Bent, R. B. and Llewellyn, S. K.: Description of the 1965-1971 ionospheric model in the definite orbit determination system (DODS), DBA Systems, Melbourne, Florida, USA, 1970.

Sheikh, N. M., Neske, E., Rawer, K., and Rebstock, C.: Comparison of peak electron densities of the F2-Layer derived from insitu measurements with CCIR predictions, Telecommunication Journal, 45-V/1978, 1978a.

Sheikh, N. M., Rawer, K., and Neske, E.: Latitudinal and Longitudinal Variations of the F2-Layer Peak Electron Density obtained from in-situ Measurement and Comparison with Predictions from the CCIR Model, URSI-XIX General Assembly, August 1978, Helsinki, Finland, 1978b. 